\title{
RESEARCH
}

Open Access

\section{Epidemiology of sepsis and septic shock in intensive care units between sepsis-2 and sepsis-3 populations: sepsis prognostication in intensive care unit and emergency room (SPICE-ICU)}

Toshikazu Abe ${ }^{1,2,3^{*}}$, Kazuma Yamakawa ${ }^{4}$, Hiroshi Ogura ${ }^{5}$, Shigeki Kushimoto ${ }^{6}$, Daizoh Saitoh ${ }^{7}$, Seitaro Fujishima ${ }^{8}$, Yasuhiro Otomo ${ }^{9}$, Joji Kotani ${ }^{10}$, Yutaka Umemura ${ }^{5}$, Yuichiro Sakamoto ${ }^{11}$, Junichi Sasaki ${ }^{12}$, Yasukazu Shiino ${ }^{13}$, Naoshi Takeyama ${ }^{14}$, Takehiko Tarui $^{15}$, Shin-ichiro Shiraishi ${ }^{16}$, Ryosuke Tsuruta ${ }^{17}$, Taka-aki Nakada ${ }^{18}$, Toru Hifumi $^{19}$, Akiyoshi Hagiwara ${ }^{20}$, Masashi Ueyama ${ }^{21}$, Norio Yamashita ${ }^{22}$, Tomohiko Masuno ${ }^{23}$, Hiroto lkeda ${ }^{24}$, Akira Komori ${ }^{25}$, Hiroki Iriyama ${ }^{25}$, Satoshi Gando ${ }^{26,27}$ and for the JAAM SPICE Study Group

\footnotetext{
Abstract

Background: Diagnosing sepsis remains difficult because it is not a single disease but a syndrome with various pathogen- and host factor-associated symptoms. Sepsis-3 was established to improve risk stratification among patients with infection based on organ failures, but it has been still controversial compared with previous definitions. Therefore, we aimed to describe characteristics of patients who met sepsis-2 (severe sepsis) and sepsis-3 definitions.

Methods: This was a multicenter, prospective cohort study conducted by 22 intensive care units (ICUs) in Japan. Adult patients ( $\geq 16$ years) with newly suspected infection from December 2017 to May 2018 were included. Those without infection at final diagnosis were excluded. Patient's characteristics and outcomes were described according to whether they met each definition or not.

\footnotetext{
* Correspondence: abetoshi111@gmail.com

'Department of Health Services Research, Faculty of Medicine, University of Tsukuba, 1-1-1 Tennodai, Tsukuba, Ibaraki 305-8577, Japan

${ }^{2}$ Health Services Research and Development Center, University of Tsukuba, Tsukuba, Japan

Full list of author information is available at the end of the article
}

(c) The Author(s). 2020 Open Access This article is licensed under a Creative Commons Attribution 4.0 International License, which permits use, sharing, adaptation, distribution and reproduction in any medium or format, as long as you give appropriate credit to the original author(s) and the source, provide a link to the Creative Commons licence, and indicate if changes were made. The images or other third party material in this article are included in the article's Creative Commons licence, unless indicated otherwise in a credit line to the material. If material is not included in the article's Creative Commons licence and your intended use is not permitted by statutory regulation or exceeds the permitted use, you will need to obtain permission directly from the copyright holder. To view a copy of this licence, visit http://creativecommons.org/licenses/by/4.0/. The Creative Commons Public Domain Dedication waiver (http://creativecommons.org/publicdomain/zero/1.0/) applies to the data made available in this article, unless otherwise stated in a credit line to the data. 
(Continued from previous page)

Results: In total, 618 patients with suspected infection were admitted to 22 ICUs during the study, of whom 530 (85.8\%) met the sepsis-2 definition and 569 (92.1\%) met the sepsis-3 definition. The two groups comprised different individuals, and 501 (81.1\%) patients met both definitions. In-hospital mortality of study population was 19.1\%. Inhospital mortality among patients with sepsis-2 and sepsis-3 patients was comparable (21.7\% and 19.8\%, respectively). Patients exclusively identified with sepsis-2 or sepsis-3 had a lower mortality (17.2\% vs. 4.4\%, respectively). No patients died if they did not meet any definitions. Patients who met sepsis-3 shock definition had higher in-hospital mortality than those who met sepsis-2 shock definition.

Conclusions: Most patients with infection admitted to ICU meet sepsis-2 and sepsis-3 criteria. However, in-hospital mortality did not occur if patients did not meet any criteria. Better criteria might be developed by better selection and combination of elements in both definitions.

Trial registration: UMIN000027452

Keywords: Intensive care unit, Sepsis, In-hospital mortality

\section{Key points}

- The majority of patients with suspected infection admitted to the ICU met sepsis-2 and sepsis-3 definitions.

- In-hospital mortality did not occur if patients did not meet any sepsis definitions.

\section{Background}

Sepsis is an aberrant or dysregulated host response resulting in organ dysfunctions and is different from infection [1]. It is not a single disease but a syndrome exhibiting with various symptoms caused by pathogens and host factors. Sepsis should be immediately recognized because it is the primary cause of death from infection, especially if not diagnosed and treated promptly. Sepsis-2 has high sensitivity [2] but captures mild infection and not infectious diseases. Sepsis-3 was established to improve risk stratification among patients with a suspected infection focusing on organ failures [1].

When considering previous studies about the diagnosis and taxonomy of sepsis to date $[1,3,4]$, nearly all of them just defined sepsis as cases of high mortality due to infectious diseases. Sepsis studies may be controversial because they were unable to differentiate an aberrant or dysregulated host response itself from infection. The definitions of sepsis- 2 and sepsis- 3 have still been inadequate to accurately capture sepsis. Therefore, both definitions may have misclassified patients with sepsis as patients with infectious diseases. Although the true nature of sepsis remains to be identified, we should clearly know what the definitions of sepsis- 2 and sepsis- 3 indicate because different definitions could change its epidemiology to identify the clinical care, future research, and healthcare planning. Such information would facilitate the definition criteria of the next sepsis. Therefore, this study aimed to describe characteristics of patients who met sepsis-2 and sepsis-3 definitions.

\section{Methods \\ Design and setting}

This multicenter, prospective cohort study was conducted in an intensive care unit (ICU) subset of the Japanese Association for Acute Medicine Sepsis Prognostication in Intensive Care Unit and Emergency Room (JAAM SPICE-ICU), including 22 ICUs in Japan from December 2017 to May 2018.

\section{Participants}

Adult patients ( $\geq 16$ years) with newly suspected infection were included. Suspected infection was defined by the administration of any kind of antibiotic, and thereby a culture of body fluids or imaging should be conducted to identify the infectious pathogen. All patients were admitted to the ICUs in study hospitals. Exclusion criteria included patients who were not transferred from other hospitals and those without infection at the final diagnosis.

\section{Data collection}

Data were extracted from the SPICE database, compiled by SPICE investigators. Collected variables included relevant patient information, such as demographics, comorbidities, degree of clinical frailty, vital signs, and site of infection. In-hospital mortality was identified as the primary outcome. Secondary outcomes were ventilator-free days (VFD), intensive care unit-free days (ICU-free days), length of hospital stay (LOS), and condition at discharge. Data collection was conducted as part of the clinical routine workup. SPICE site investigators recorded all data throughout the patient's hospital stays. If case of missing data, the SPICE committee requested a reconfirmation of data extraction from SPICE investigators.

\section{Data definitions}

Sepsis-2 was defined as having a suspected site of infection, $\geq 2$ systemic inflammatory response syndrome 


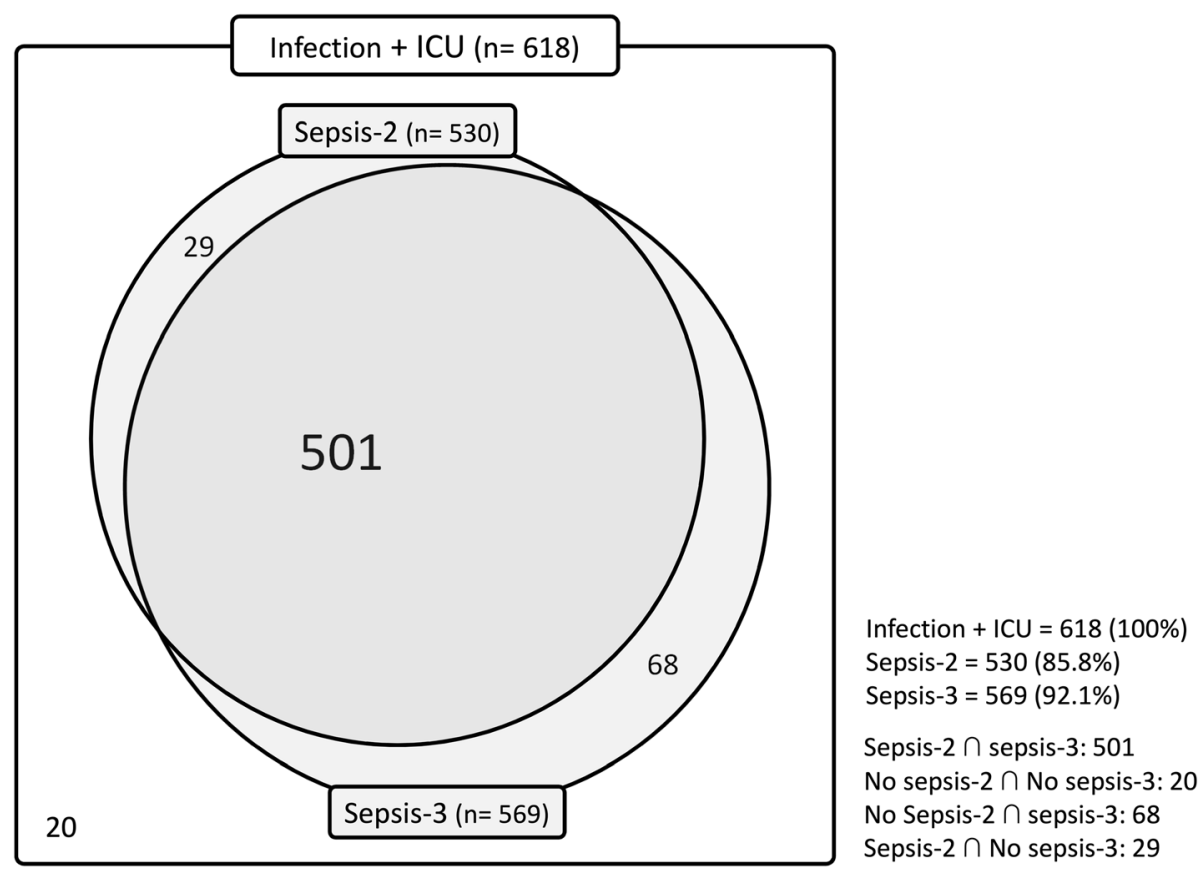

Fig. 1 Taxonomy and in-hospital mortality among patients with infection admitted to the intensive care unit

criteria (SIRS) [5] and $\geq 1$ organ dysfunction criteria [6]. Severe sepsis was actually defied as sepsis- 2 according to the sepsis-2 definition [3]. Sepsis-3 was defined as having a suspected site of infection and organ dysfunction (an acute change in the total sequential organ failure assessment (SOFA) score of $\geq 2$ points consequent to the infection) [1]. Regarding shock, sepsis-2 and sepsis-3 shocks were defined according to the sepsis-2 [6] and sepsis-3 definitions, respectively [1] (Supplemental file 1). Frailty was defined according to the Clinical Frailty Scale (CFS), an easy and intuitive determinable categorization tool based on simple visual descriptions [7]. Patients' status for CFS before hospital admission was obtained from patients themselves or their relatives. Infection sites at final diagnosis included the lung, intraabdominal, urinary tract, soft tissue, central nervous system (CNS), osteoarticular, endocardium, wound, catheter-related, implant device-related, others, or unidentified infections. The diagnosis of the infection site was recorded at discharge. Acute physiology and chronic health evaluation II (APACHE II) score was calculated at the initial examination instead of the worst data within $24 \mathrm{~h}$. If APACHE II score was missing, zero was used instead of missing data. SOFA score was calculated similarly as the APACHE II score. VFD was defined as the number of days within the first 28 days postadmission that the patient can breathe without a ventilator. The VFD of patients who died during the study period was set as zero. ICU-free days were calculated similarly with VFD. Status at discharge was categorized as home, transfer to another facility (including longterm care and nursing homes), or death.

\section{Analysis}

Patients with infection in ICUs were compared according to whether they met sepsis- 2 or sepsis-3 definition. Patients were divided into five groups: sepsis-2; sepsis-3; sepsis-2 and sepsis-3; sepsis-2 and no sepsis-3; no sepsis-2 and sepsis-3; and no sepsis-2 and no sepsis-3. Descriptive statistics included proportions for categorical variables, and medians (interquartile range $[\mathrm{IQR}]$ ) of continuous variables were calculated because not all variables were normally distributed. A few missing data was considered missing randomly. No assumptions were made on these data. All statistical analyses were performed using the Stata software version 15.1 (StataCorp, TX, USA).

\section{Results}

A total of 618 patients with suspected infection admitted to 22 ICUs during the study period were included in this study. Among them, $530(85.8 \%)$ patients had sepsis-2, and 569 (92.1\%) had sepsis-3; most patients were overlapped; however, patients with sepsis-2 and sepsis-3 were different individuals. A total of 501 (81.1\%) met both definitions, and 29 exclusively met the sepsis-2 (only 
Table 1 Case mix characteristics for admissions to ICU with suspected infection (by sepsis-2 and sepsis-3 definitions)

\begin{tabular}{|c|c|c|c|c|c|c|c|}
\hline & & Sepsis-2 & Sepsis-3 & $\begin{array}{l}\text { Sepsis-2 and } \\
\text { sepsis-3 }\end{array}$ & $\begin{array}{l}\text { Sepsis-2 and no } \\
\text { sepsis-3 }\end{array}$ & $\begin{array}{l}\text { No sepsis-2 and } \\
\text { sepsis-3 }\end{array}$ & $\begin{array}{l}\text { No sepsis-2 and no } \\
\text { sepsis-3 }\end{array}$ \\
\hline Variables & & $n=530$ & $n=569$ & $n=501$ & $n=29$ & $n=68$ & $n=20$ \\
\hline Age at admission (ye & & $72(60-81)$ & $72(60-81)$ & $73(61-81)$ & $69(57-75)$ & $66(57-81)$ & $70(55-79)$ \\
\hline Gender (male) & & $299(56.4)$ & $318(55.9)$ & $281(56.1)$ & $18(62.1)$ & $37(54.4)$ & $13(65.0)$ \\
\hline $\mathrm{BMI}\left(\mathrm{kg} / \mathrm{m}^{2}\right)$ & & $\begin{array}{l}21.8(19.4- \\
24.3)\end{array}$ & $\begin{array}{l}21.9(19.3- \\
24.3)\end{array}$ & $\begin{array}{l}21.9(19.5- \\
24.3)\end{array}$ & $21.3(19.3-24.2)$ & $21.8(18.3-24.2)$ & $23.4(19.2-26.3)$ \\
\hline Admission source & Emergency department & $304(57.4)$ & $317(55.7)$ & $285(56.9)$ & $19(65.5)$ & $32(47.1)$ & $14(70.0)$ \\
\hline & $\begin{array}{l}\text { Non-ED (hospital/dept. } \\
\text { transfers) }\end{array}$ & $204(38.5)$ & $230(40.4)$ & $200(39.9)$ & $4(13.8)$ & $30(44.1)$ & $2(10.0)$ \\
\hline & Intensive care unit & $22(4.2)$ & $22(3.9)$ & $16(3.2)$ & $6(20.7)$ & $6(8.8)$ & $4(20.0)$ \\
\hline Charlson & 0 & $156(29.4)$ & $169(29.7)$ & $147(29.3)$ & $9(31.0)$ & $22(32.3)$ & $8(40.0)$ \\
\hline & $1-2$ & $227(42.8)$ & $249(43.8)$ & $214(42.7)$ & $13(44.8)$ & $35(51.5)$ & $8(40.0)$ \\
\hline & $3-4$ & $104(19.6)$ & $105(18.5)$ & $99(19.8)$ & $5(17.2)$ & $6(8.8)$ & $2(10.0)$ \\
\hline & $>4$ & $43(8.1)$ & $46(8.1)$ & $41(8.2)$ & $2(6.9)$ & $5(7.4)$ & $2(10.0)$ \\
\hline Clinical Frailty Scale & Fit (CFS 1-3) & $269(50.9)$ & $289(50.8)$ & $249(49.8)$ & $20(71.4)$ & $40(58.8)$ & $12(60.0)$ \\
\hline & Pre-frail (CFS 4) & $90(17.0)$ & $100(17.6)$ & $89(17.8)$ & $1(3.6)$ & $11(16.2)$ & $4(20.0)$ \\
\hline & Frail (CFS 5-9) & $169(32.0)$ & 179 (31.5) & $162(32.4)$ & $87(25.0)$ & $17(25.0)$ & $4(20.0)$ \\
\hline Immuno-insufficienc & $y$ at APACHE ॥ & $110(20.8)$ & $111(19.6)$ & $101(20.2)$ & $9(31.0)$ & $10(14.9)$ & $3(15.0)$ \\
\hline Baseline SOFA for se & bsis-3 & $0(0-1)$ & $0(0-1)$ & $0(0-1)$ & $4(0-8)$ & $0(0-0)$ & $1(0-3)$ \\
\hline Glasgow Coma Scale & & $12(7-14)$ & $12(7-15)$ & $11(7-14)$ & $14(11-15)$ & $14(10-15)$ & $15(14-15)$ \\
\hline Intubated & & $225(42.6)$ & $226(39.7)$ & $212(42.3)$ & $13(44.8)$ & $14(20.6)$ & $5(26.3)$ \\
\hline Systolic blood pressu & re $(\mathrm{mmHg})$ & $\begin{array}{l}102(82- \\
126)\end{array}$ & $\begin{array}{l}105(84- \\
128)\end{array}$ & $101(82-125)$ & $122(107-143)$ & $122(107-141)$ & $123(111-143)$ \\
\hline Heat rate (/min) & & $\begin{array}{l}107(90- \\
124)\end{array}$ & $\begin{array}{l}107(89- \\
122)\end{array}$ & $108(91-124)$ & $93(82-108)$ & $90(80-108)$ & $90(79-106)$ \\
\hline Respiratory rate (/mir & & $24(19-30)$ & $24(19-30)$ & $24(19-30)$ & $19(17-24)$ & $20(18-26)$ & $20(19-25)$ \\
\hline Body temperature $\left(^{\circ}\right.$ & & $\begin{array}{l}37.3(36.5- \\
38.5)\end{array}$ & $\begin{array}{l}37.4(36.5- \\
38.4)\end{array}$ & $\begin{array}{l}37.4(36.5- \\
38.5)\end{array}$ & $37.1(36.3-38.5)$ & $37.5(36.8-38.3)$ & $37.7(36.7-38.3)$ \\
\hline Lactate (mmol/L) & & $\begin{array}{l}2.9(1.7- \\
4.9)\end{array}$ & $\begin{array}{l}2.7(1.5- \\
4.7)\end{array}$ & $3.1(1.7-5.1)$ & $2.1(1.4-2.9)$ & $1.2(0.9-1.8)$ & $1.0(0.9-1.6)$ \\
\hline Positive blood cultur & & $252(50.0)$ & $261(48.4)$ & $245(51.3)$ & $7(26.9)$ & $16(23.5)$ & $4(23.5)$ \\
\hline Site of infection & Lung & $200(37.7)$ & $208(36.6)$ & $192(38.3)$ & $8(27.6)$ & $16(23.5)$ & $8(40.0)$ \\
\hline & Abdomen & $111(20.9)$ & $119(20.9)$ & $101(20.2)$ & $10(34.5)$ & $18(26.5)$ & $2(10.0)$ \\
\hline & Urinary tract & $91(17.2)$ & $101(17.8)$ & $89(17.8)$ & $2(6.9)$ & $12(17.7)$ & $3(15.0)$ \\
\hline & Soft tissue & $65(12.3)$ & $70(12.3)$ & $60(12.0)$ & $5(17.2)$ & $10(14.7)$ & $6(30.0)$ \\
\hline & Central nervous system & $11(2.1)$ & $13(2.3)$ & $10(2.0)$ & $1(3.5)$ & $3(4.4)$ & $1(5.0)$ \\
\hline & Intravenous catheter & $4(0.8)$ & $4(0.7)$ & $4(0.8)$ & 0 & 0 & 0 \\
\hline & Osteoarticular & $4(0.8)$ & $6(1.1)$ & $4(0.8)$ & 0 & $2(2.9)$ & 0 \\
\hline & Endocardium & $1(0.2)$ & $1(0.2)$ & $1(0.2)$ & 0 & 0 & 0 \\
\hline & Wound & $5(0.9)$ & $5(0.9)$ & $4(0.8)$ & $1(3.5)$ & $1(1.5)$ & 0 \\
\hline & Implant device & $2(0.4)$ & $2(0.4)$ & $2(0.4)$ & 0 & 0 & 0 \\
\hline & Other & $14(2.6)$ & $16(2.8)$ & $13(2.6)$ & $1(3.5)$ & $3(4.4)$ & 0 \\
\hline & Unidentified & $22(4.2)$ & $24(4.2)$ & $21(4.2)$ & $1(3.5)$ & $3(4.4)$ & 0 \\
\hline $\begin{array}{l}\text { Organ dysfunction } \\
\text { on arrival }\end{array}$ & $\begin{array}{l}\text { Acute lung injury with } \\
\text { pneumonia }\end{array}$ & $127(24.0)$ & $124(21.8)$ & $124(24.8)$ & $3(10.3)$ & 0 & 0 \\
\hline & $\begin{array}{l}\text { Acute lung injury without } \\
\text { pneumonia }\end{array}$ & $96(18.2)$ & $99(17.5)$ & $94(18.8)$ & $2(7.1)$ & $5(7.4)$ & 0 \\
\hline
\end{tabular}


Table 1 Case mix characteristics for admissions to ICU with suspected infection (by sepsis-2 and sepsis-3 definitions) (Continued)

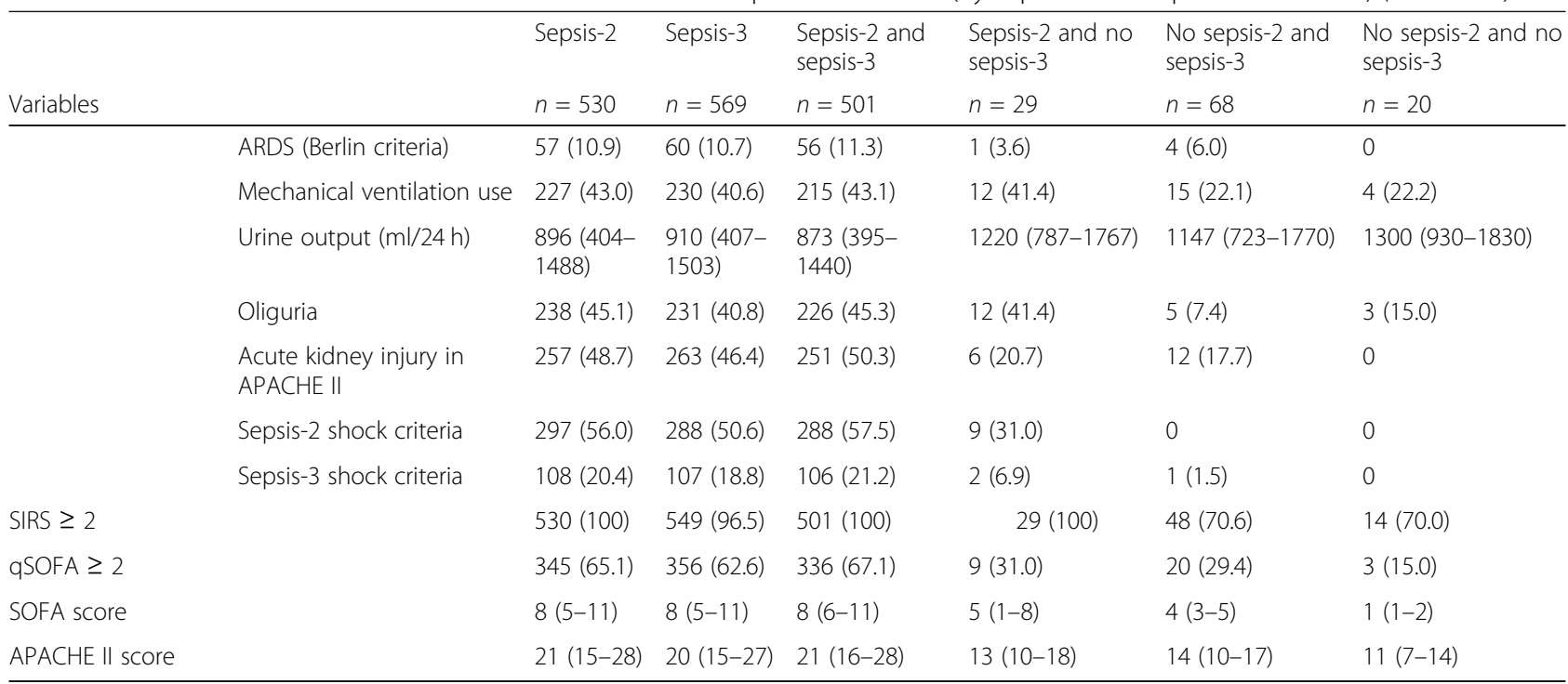

Reported counts (proportions) for categorical variables and median (interquartile range) for continuous variables

Missing; BMI = 5; Clinical Frailty Scale = 2; Immuno-insufficiency at APACHE II = 3; Intubation = 1; Systolic blood pressure = 2; Heart rate = 1; Body temperature = 1; Lactate = 14; Blood culture = 36; Acute lung injury without pneumonia = 3; ARDS = 9; Mechanical ventilation use = 4; Urine output = 36; Oliguria = 2; Acute kidney injury at APACHE $\|=2$

$B M I$ body mass index, ED emergency department, ICU intensive care unit, CFS Clinical Frailty Scale, APACHE II Acute physiology and chronic health evaluation II, SOFA sequential (sepsis-related) organ failure assessment, WBC white blood cell, ARDS acute respiratory distress syndrome, SIRS systemic inflammatory response syndrome

sepsis-2), and 68 exclusively met the sepsis-3 (only sepsis-3) definition. A total of 20 patients did not meet either of the definitions (Fig. 1). Majority of patients were admitted to the ICUs directly from the emergency departments (EDs) (56.6\%). A total of 592 (95.8\%) patients were positive for SIRS, and 368 (59.6\%) were positive for qSOFA. A total of 297 (48.1\%) patients met the sepsis-2 shock criteria, and 109 (17.6\%) met the sepsis-3 shock criteria.

Table 1 shows characteristics of patients with infection in ICUs according to sepsis definitions. The distributions of baseline characteristics such as age, sex, and comorbidities were comparable between sepsis-2 and sepsis-3. The baseline SOFA score for the sepsis- 2 only group was 4 (IQR, 0-8) although the baseline SOFA for other groups was 0 or 1 . Lactate and blood culture positivity were lower if they did not meet the definitions. The SOFA score was lower if they did not meet any definitions. The trend in pathogens and antibiotics according to sepsis definitions was also nonspecific; however, blood culture positivity was lower if patients did not meet any definitions (sepsis-2 or sepsis-3), and carbapenem was more frequently used in patients who met any definitions (Table 2).

In-hospital mortality of study population was $19.1 \%$. In-hospital mortality among patients with sepsis- 2 and sepsis- 3 patients was comparable $(21.7 \%$ and $19.8 \%$, respectively) (Table 3). Patients exclusively identified by sepsis-2 or sepsis-3 had a lower mortality $(17.2 \%$ vs.
$4.4 \%$, respectively). No patients died if they did not meet any definitions. Patients who met sepsis- 3 shock criteria had higher in-hospital mortality than those who met sepsis-2 shock criteria.

\section{Discussion}

\section{Summary}

Characteristics and in-hospital mortality were compared according to sepsis- 2 and sepsis- 3 definitions in this prospective observational cohort of ICU patients. Almost all patients presenting to ICU with infection fulfill both definitions, but what each definition identified is different. However, in-hospital mortality was zero if patients did not meet any definitions. Better criteria might be developed by better selection and combination of elements in both definitions.

\section{Comparison with previous studies}

Nearly all patients admitted to ICU with suspected infection fulfill both definitions in our study as well as in previous studies $[8,9]$. In a retrospective cohort study of ICUs in England, with similar setting as in our study, sepsis- 2 and sepsis- 3 definitions identified similar populations (92\% overlapped), which is consistent with that of our study ( $81 \%$ overlapped). Actually, $95 \%$ of patients overlapped if sepsis-3 definition was evaluated in sepsis2 (severe sepsis) population in our cohort. Their severity scores, such as the SOFA, were derived from an estimation such as a receipt of organ support and could have 
Table 2 Pathogens and antibiotics according to the sepsis definitions

\begin{tabular}{|c|c|c|c|c|c|c|c|}
\hline \multirow{2}{*}{ Variables } & & \multirow{2}{*}{$\begin{array}{l}\text { Sepsis-2 } \\
n=530\end{array}$} & \multirow{2}{*}{$\begin{array}{l}\text { Sepsis-3 } \\
n=569\end{array}$} & \multirow{2}{*}{$\begin{array}{l}\text { Sepsis-2 and } \\
\text { sepsis-3 } \\
n=501\end{array}$} & \multirow{2}{*}{$\begin{array}{l}\text { Sepsis-2 and no } \\
\text { sepsis-3 } \\
n=29\end{array}$} & \multirow{2}{*}{$\begin{array}{l}\text { No sepsis-2 and } \\
\text { sepsis-3 } \\
n=68\end{array}$} & \multirow{2}{*}{$\begin{array}{l}\text { No sepsis-2 } \\
\text { and no sepsis-3 } \\
n=20\end{array}$} \\
\hline & & & & & & & \\
\hline \multicolumn{8}{|l|}{ Microbiology of blood cultures } \\
\hline \multirow[t]{3}{*}{ Gram-negative } & E. coli & $\begin{array}{l}84 \\
(15.9)\end{array}$ & $\begin{array}{l}89 \\
(15.6)\end{array}$ & $84(16.8)$ & 0 & $5(7.4)$ & 0 \\
\hline & Klebsiella & $35(6.6)$ & $34(6.0)$ & $34(6.8)$ & $1(3.5)$ & 0 & $1(5.0)$ \\
\hline & Pseudomonas & $2(0.4)$ & $2(0.4)$ & $2(0.4)$ & 0 & 0 & 0 \\
\hline \multirow[t]{5}{*}{ Gram-positive } & Staphylococci & $\begin{array}{l}62 \\
(11.7)\end{array}$ & $\begin{array}{l}66 \\
(11.6)\end{array}$ & $60(12.0)$ & $2(6.9)$ & $6(8.8)$ & 0 \\
\hline & Streptococci & $43(8.1)$ & $45(7.9)$ & $42(8.4)$ & $1(3.5)$ & $3(4.4)$ & $1(5.0)$ \\
\hline & MRSA & $5(0.9)$ & $6(1.1)$ & $5(1.0)$ & 0 & $1(1.5)$ & 0 \\
\hline & Enterococcus & $12(2.3)$ & $12(2.1)$ & $12(2.4)$ & 0 & 0 & 0 \\
\hline & Anaerobic & $14(2.6)$ & $12(2.1)$ & $12(2.4)$ & $2(6.9)$ & 0 & 0 \\
\hline Fungi & & $2(0.4)$ & $2(0.4)$ & $2(0.4)$ & 0 & 0 & 0 \\
\hline \multicolumn{8}{|l|}{ Antibiotics } \\
\hline Penicillin derivative (PCG, ABPC, ABPC/MCIPC) & & $21(4.0)$ & $27(4.8)$ & $21(4.2)$ & 0 & $6(8.8)$ & $1(5.0)$ \\
\hline Ampicillin/sulbactam & & $\begin{array}{l}69 \\
(13.0)\end{array}$ & $\begin{array}{l}77 \\
(13.5)\end{array}$ & $66(13.2)$ & $3(10.3)$ & $11(16.2)$ & $7(35.0)$ \\
\hline $\mathrm{PIPC} / \mathrm{TAZ}$ & & $\begin{array}{l}73 \\
(13.8)\end{array}$ & $\begin{array}{l}73 \\
(12.8)\end{array}$ & $64(12.8)$ & $9(31.0)$ & $9(13.2)$ & $6(30.0)$ \\
\hline First generation cephalosporin & & $11(2.1)$ & $11(2.0)$ & $9(1.8)$ & $2(6.9)$ & $2(2.9)$ & $3(15.0)$ \\
\hline $\begin{array}{l}\text { Second generation cephalosporin (CTM, CMZ, } \\
\text { FMOX) }\end{array}$ & & $13(2.5)$ & $17(3.0)$ & $11(2.2)$ & $2(6.9)$ & $6(8.8)$ & 0 \\
\hline Third generation cephalosporin (CTX, CPZ, CTRX) & & $\begin{array}{l}60 \\
(11.3)\end{array}$ & $\begin{array}{l}67 \\
(11.8)\end{array}$ & $57(11.4)$ & $3(10.3)$ & $10(14.7)$ & $1(5.0)$ \\
\hline $\begin{array}{l}\text { Third generation cephalosporin against } \\
\text { pseudomonas }\end{array}$ & & $3(0.6)$ & $3(0.5)$ & $3(0.6)$ & 0 & 0 & 0 \\
\hline $\begin{array}{l}\text { Fourth generation cephalosporin against } \\
\text { pseudomonas }\end{array}$ & & $30(5.7)$ & $29(5.1)$ & $29(5.8)$ & $1(3.5)$ & 0 & 0 \\
\hline Carbapenem & & $\begin{array}{l}279 \\
(52.6)\end{array}$ & $\begin{array}{l}291 \\
(51.1)\end{array}$ & $268(53.5)$ & $11(37.9)$ & $23(33.8)$ & $3(15.0)$ \\
\hline Aminoglycoside & & 0 & 0 & 0 & 0 & 0 & 0 \\
\hline Quinolone & & $32(6.0)$ & $35(6.2)$ & $32(6.4)$ & 0 & $3(4.4)$ & 0 \\
\hline Tetracycline & & $3(0.6)$ & $3(0.5)$ & $3(0.6)$ & 0 & 0 & 0 \\
\hline Macrolide & & $29(5.5)$ & $29(5.1)$ & $29(5.8)$ & 0 & 0 & 0 \\
\hline Metronidazole & & $18(3.4)$ & $18(3.2)$ & $18(3.6)$ & 0 & 0 & 0 \\
\hline CLDM & & $23(4.3)$ & $24(4.2)$ & $20(4.0)$ & $3(10.3)$ & $4(5.9)$ & $1(5.0)$ \\
\hline Vancomycin & & $\begin{array}{l}81 \\
(15.3)\end{array}$ & $\begin{array}{l}80 \\
(14.1)\end{array}$ & $75(15.0)$ & $6(20.7)$ & $5(7.4)$ & $4(20.0)$ \\
\hline $\begin{array}{l}\text { Other anti-methicillin-resistant staphylococcus } \\
\text { aureus drugs }\end{array}$ & & $45(8.5)$ & $48(8.4)$ & $45(9.0)$ & 0 & $3(4.4)$ & 0 \\
\hline Antifungus & & $28(5.3)$ & $30(5.3)$ & $28(5.6)$ & 0 & $2(2.9)$ & $1(5.0)$ \\
\hline Others (ST, CLL, FOM, SBT/CPZ, AMPC) & & $10(1.9)$ & $11(1.9)$ & $10(2.0)$ & 0 & $1(1.5)$ & $1(5.0)$ \\
\hline
\end{tabular}

Reported counts (proportions)

$I C U$ intensive care unit, $P C G$ penicillin G, ABPC ampicillin, ABPC/MCIPC ampicillin/cloxacillin, PIPC/TAZ tazobactam/piperacillin, CTM cefotiam, CMZ cefmetazole, FMOX flomoxef, CTX cefotaxime, CPZ cefoperazone, CTRX ceftriaxone, CLDM clindamycin, ST sulfamethoxazole-trimethoprim, CLL cefaclor, FOM fosfomycin, SBT/CPZ sulbactam/cefoperazone, AMPC amoxicillin, MRSA methicillin-resistant Staphylococcus aureus

been over- or underestimated in the study [9]. Another study also virtually calculated the SOFA score, even though it is one of the most important elements in sepsis-3 definition [8]. Previous studies reported some variations of epidemiology by data sources, data acquisition timing, and interpretation of organ failure criteria in sepsis criteria $[8$,
$10,11]$. Our prospective study was designed to compare sepsis-2 and sepsis-3 among patients with suspected infection and directly confirmed results of previous studies $[8$, 9]. However, these minor variations in the precise interpretation of definitions may have not affect characteristic and mortality differences, especially in the ICU setting [8]. 
Table 3 Outcomes according to the sepsis definitions

\begin{tabular}{|c|c|c|c|c|c|c|c|}
\hline & & Sepsis-2 & Sepsis-3 & $\begin{array}{l}\text { Sepsis-2 and } \\
\text { sepsis-3 }\end{array}$ & $\begin{array}{l}\text { Sepsis-2 and no } \\
\text { sepsis-3 }\end{array}$ & $\begin{array}{l}\text { No sepsis-2 and } \\
\text { sepsis-3 }\end{array}$ & $\begin{array}{l}\text { No sepsis-2 and no } \\
\text { sepsis-3 }\end{array}$ \\
\hline Outcomes & & $n=530$ & $n=569$ & $n=501$ & $n=29$ & $n=68$ & $n=20$ \\
\hline \multirow[t]{4}{*}{$\begin{array}{l}\text { In-hospital } \\
\text { mortality }\end{array}$} & All & $\begin{array}{l}114 / 526 \\
(21.7)\end{array}$ & $\begin{array}{l}112 / 565 \\
(19.8)\end{array}$ & 109/497 (21.9) & $5 / 29(17.2)$ & $3 / 68(4.4)$ & 0/19 (0) \\
\hline & $\begin{array}{l}\text { Shock Sepsis-2 } \\
\text { Criteria }\end{array}$ & $\begin{array}{l}80 / 295 \\
(27.1)\end{array}$ & $\begin{array}{l}78 / 286 \\
(27.3)\end{array}$ & 78/286 (27.3) & 2/9 (22.2) & 0 & 0 \\
\hline & $\begin{array}{l}\text { Shock Sepsis-3 } \\
\text { Criteria }\end{array}$ & $\begin{array}{l}36 / 107 \\
(33.6)\end{array}$ & $\begin{array}{l}36 / 106 \\
(34.0)\end{array}$ & 36/105 (34.3) & $0 / 2(0)$ & $0 / 1(0)$ & 0 \\
\hline & Patients from ED & $\begin{array}{l}57 / 303 \\
(18.8)\end{array}$ & $\begin{array}{l}55 / 316 \\
(17.4)\end{array}$ & $54 / 284(19.0)$ & 3/19 (15.8) & 1/32 (3.1) & 0/13 (0) \\
\hline \multirow[t]{2}{*}{$\begin{array}{l}\text { Survivor } \\
\text { dispositions }\end{array}$} & Home & $\begin{array}{l}152 / 526 \\
(28.9)\end{array}$ & $\begin{array}{l}171 / 565 \\
(30.3)\end{array}$ & $140 / 497(28.2)$ & $12 / 29(41.4)$ & $31 / 68(45.6)$ & 10/19 (52.6) \\
\hline & Transfer & $\begin{array}{l}260 / 526 \\
(49.4)\end{array}$ & $\begin{array}{l}282 / 565 \\
(49.9)\end{array}$ & 248/497 (49.9) & $12 / 29(41.4)$ & $34 / 68(50.0)$ & $9 / 19(47.4)$ \\
\hline 28-day mortality & & $\begin{array}{l}98 / 503 \\
(19.5)\end{array}$ & $\begin{array}{l}99 / 536 \\
(18.5)\end{array}$ & 96/474 (20.3) & 2/29 (6.9) & 3/62 (4.8) & 0/19 (0) \\
\hline ICU-free days & & $13(0-21)$ & $15(0-21)$ & $13(0-21)$ & $19(2-24)$ & $22(15-25)$ & $24(23-26)$ \\
\hline $\begin{array}{l}\text { Ventilator-free } \\
\text { days }\end{array}$ & & $20(0-28)$ & $21(0-28)$ & $20(0-26)$ & $21(0-28)$ & $27(22-28)$ & $28(28-28)$ \\
\hline $\begin{array}{l}\text { Length of hospital } \\
\text { stay }\end{array}$ & & $25(11-46)$ & $24(12-45)$ & $25(11-46)$ & $31(12-47)$ & $20(12-41)$ & $11(5-25)$ \\
\hline
\end{tabular}

Reported counts (proportions) for categorical and median (interquartile range) for continuous variables

Missing: In-hospital mortality = 5; 28-day mortality = 34; ICU-free days = 34; Ventilator-free days = 34; Length of hospital stay = 5 ICU intensive care unit, ED emergency department

Patients exclusively identified with sepsis-2 or sepsis-3 had different characteristics when compared to patients with both sepsis- 2 and sepsis-3. Although a total of $63 \%$ patients were diagnosed with sepsis-3 using qSOFA, only $29 \%$ of patients with sepsis-2(-)/sepsis-3(+) were diagnosed with sepsis- 3 using qSOFA. Patients exclusively identified with sepsis- 2 or sepsis-3 presumably included those who have clinically unmeasured features such as vague symptoms [12]. Patients who meet only one definition may need more attention because their symptoms were not prominent. Since sepsis-2 captures high level of inflammation, a patient with sepsis- 2 would still need further attention; however, a negative sepsis- 3 does not meet the current definition of sepsis. In-hospital mortality differed by approximately four times between the sepsis-2(+)/sepsis-3(-) group and the sepsis-2(-)/sepsis$3(+)$ group $(17 \%$ vs. $4 \%)$. Although the number of patients was small, judging by one definition alone may cause misclassification of poor outcome patients who may be identified by the other definition. However, since they were actually in the ICU, the physician did not misclassify the patients. This highlights the limitations of both the definitions.

\section{Possible explanations and implications}

Since sepsis-2 does not include an increased acute SOFA score from the baseline, any chronic organ failure may possibly be regarded as an acute organ failure. SOFA score was not identified even though sepsis- 2 was defined based on this score, except for chronic organ failures. In our cohort, 96 (16\%) patients had "not available" (NA) sepsis-3 baseline SOFA, which was indicated as zero according to the sepsis-3 definition, although all data of chronic organ failures were tried to obtain. Therefore, a number of patients with unknown chronic organ failures at baseline should have been included in those with acute organ failure in any definitions. Either way, the sepsis-3 definition has become more clinically objectively understandable than the sepsis-2 definition. Moreover, the sepsis-3 definition was originally easier to evaluate than the sepsis-2 definition.

Excluding SIRS as the starting point for sepsis-3 did not affect the incidence as majority of patients with organ failures also tend to have SIRS. The sepsis-3 shock was associated with a higher risk of death than sepsis-2 [13], because the sepsis-3 shock requires the presence of elevated serum lactate levels in addition to fluidresistant hypotension [14]. The problem of sepsis diagnosis has been a little arbitrary, with differences in epidemiology. The sepsis-3 definition may be advantageous because it may increase the comparability of sepsis incidence and related mortality among studies by possibly reducing the subjective interpretation [8]. A consistent diagnosis of sepsis and septic shock between institutions should be considered not only for research purposes but also for quality measurement. 
Generally, severity scores such as SOFA were better used for clinical research and quality measurement rather than risk assessment. Therefore, the definition of sepsis has undoubtedly dramatically advanced the research due to enhanced medical research efficiency when agreed disease and outcome definitions are used. However, definitions are still insufficient and have not been used beyond as tools of research and quality measurement.

When a patient was admitted to ICU due to an infection, his or her mortality rate was approximately $20 \%$ in this cohort. This will make little contribution even if new criteria are used because $20 \%$ is one of the highest mortality in ICU diseases. The advancement of sepsis definitions may lead to the concept that similar conditions were caused by infections, despite the different backgrounds and triggers. However, risk stratifications and predictions should be investigated in detail in the future. For example, the upgrade or downgrade type of sepsis should be assessed based on the immune response, a subgroup for site of infection, or a phenotype of treatment responsiveness. "One size fits all approach" has reached its limits.

\section{Limitations}

This study has several limitations. First, organ failure data before the ICU admission were missing in some patients, which was also noticed in the original sepsis-3 study [1]. Second, regarding to APACHE II score, data at initial diagnosis were used instead of the worst data within $24 \mathrm{~h}$ of ICU admission because of availability. This may have led to underestimation of the severity of patient conditions. Third, missing data were indicated as zero in the APACHE II and SOFA scores, if some elements were missing. This would have been used to identify any underestimation of the variance of patient's severity. However, effects of missing data should be small because missing data of elements were few.

\section{Conclusions}

A majority of the patients who were admitted to the ICU with suspected infection met sepsis-2 and sepsis-3 definitions. In-hospital mortality was indicated as zero if patients did not meet any sepsis definitions.

\section{Supplementary information}

Supplementary information accompanies this paper at https://doi.org/10. 1186/s40560-020-00465-0.

Additional file 1:. Supplemental file 1

\section{Abbreviations}

SPICE-ICU: Sepsis Prognostication in Intensive Care Unit and Emergency Room; ICUs: Intensive care units; VFD: Ventilator-free days; ICU-free days: Intensive care unit-free days; LOS: Length of hospital stay; SIRS: Systemic inflammatory response syndrome criteria; SOFA: Sequential organ failure assessment; CFS: Clinical Frailty Scale; CNS: Central nervous system; APACHE II: Acute physiology and chronic health evaluation II; ED: Emergency department; NA: Not available

\section{Acknowledgements}

We thank the JAAM SPICE Study Group for the contribution to this study. We would like to thank Enago (https://www.enago.jp) for English language editing. This work was supported by JSPS KAKENHI Grant Number JP19K19376.

\section{Notation of prior abstract publication/presentation}

We will present this research at 33rd annual congress European society of intensive care medicine (ESICM) 2020.

\section{Authors' contributions}

TA contributed to the acquisition of data, conceived of and designed this study, interpreted the data, drafted the manuscript, and revised the manuscript for important intellectual content. KY contributed to the acquisition of data, conducted data cleaning, interpreted the data, and revised the manuscript for important intellectual content. All of the authors contributed to the acquisition of data, and reviewed, discussed, and approved the final manuscript.

\section{Funding}

This study was supported by the Japanese Association for Acute Medicine (2014-01).

\section{Availability of data and materials}

The datasets generated during and/or analyzed during the current study are available from the corresponding author on reasonable request.

\section{Ethics approval and consent to participate}

The study protocol was reviewed and approved by the Research Ethics Committee of all participating institutions at the Japanese Association for Acute Medicine (JAAM) SPICE study group. Given the retrospective and anonymized nature of this study in the routine care, the Ethics Committees waived the need for informed consent from the study participants. The Institutional Review Board of Hokkaido University, a leading institution in SPICE, approved this study (approval no. 016-0386).

\section{Consent for publication}

Not applicable

\section{Competing interests}

All authors declare that they have no competing interests.

\section{Author details}

${ }^{1}$ Department of Health Services Research, Faculty of Medicine, University of Tsukuba, 1-1-1 Tennodai, Tsukuba, Ibaraki 305-8577, Japan. ${ }^{2}$ Health Services Research and Development Center, University of Tsukuba, Tsukuba, Japan. ${ }^{3}$ Department of Emergency and Critical Care Medicine, Tsukuba Memorial Hospital, Tsukuba, Japan. ${ }^{4}$ Division of Trauma and Surgical Critical Care, Osaka General Medical Center, Osaka, Japan. ${ }^{5}$ Department of Traumatology and Acute Critical Medicine, Osaka University Graduate School of Medicine, Osaka, Japan. ${ }^{6}$ Division of Emergency and Critical Care Medicine, Tohoku University Graduate School of Medicine, Sendai, Japan. ${ }^{7}$ Division of Traumatology, Research Institute, National Defense Medical College, Tokorozawa, Japan. ${ }^{8}$ Center for General Medicine Education, Keio University School of Medicine, Tokyo, Japan. ${ }^{9}$ Trauma and Acute Critical Care Center, Medical Hospital, Tokyo Medical and Dental University, Tokyo, Japan.

${ }^{10}$ Division of Disaster and Emergency Medicine, Department of Surgery Related, Kobe University Graduate School of Medicine, Kobe, Japan.

${ }^{11}$ Emergency and Critical Care Medicine, Saga University Hospital, Saga, Japan. ${ }^{12}$ Department of Emergency and Critical Care Medicine, Keio University School of Medicine, Tokyo, Japan. ${ }^{13}$ Department of Acute Medicine, Kawasaki Medical School, Kurashiki, Japan. ${ }^{14}$ Advanced Critical Care Center, Aichi Medical University Hospital, Nagakute, Japan. ${ }^{15}$ Department of Trauma and Critical Care Medicine, Kyorin University School of Medicine, Tokyo, Japan. ${ }^{16}$ Department of Emergency and Critical Care Medicine, Aizu Chuo Hospital, Aizuwakamatsu, Japan. ${ }^{17}$ Advanced Medical Emergency \& Critical Care Center, Yamaguchi University Hospital, Ube, Japan.

${ }^{18}$ Department of Emergency and Critical Care Medicine, Chiba University 
Graduate School of Medicine, Chiba, Japan. ${ }^{19}$ Department of Emergency and Critical Care Medicine, St. Luke's International Hospital, Tokyo, Japan. ${ }^{20}$ Department of Emergency Medicine, Niizashiki Chuo General Hospital, Niizashiki, Japan. ${ }^{21}$ Department of Trauma, Critical Care Medicine, and Burn Center, Japan Community Healthcare Organization, Chukyo Hospital, Nagoya, Japan. ${ }^{22}$ Advanced Emergency Medical Service Center, Kurume University Hospital, Kurume, Japan. ${ }^{23}$ Department of Emergency and Critical Care Medicine, Nippon Medical School, Tokyo, Japan. ${ }^{24}$ Department of Emergency Medicine, Teikyo University School of Medicine, Tokyo, Japan. ${ }^{25}$ Department of General Medicine, Juntendo University, Tokyo, Japan. ${ }^{26}$ Division of Acute and Critical Care Medicine, Hokkaido University Graduate School of Medicine, Sapporo, Japan. ${ }^{27}$ Department of Acute and Critical Care Medicine, Sapporo Higashi Tokushukai Hospital, Sapporo, Japan.

Received: 10 May 2020 Accepted: 23 June 2020

Published online: 30 June 2020

\section{References}

1. Singer M, Deutschman CS, Seymour CW, Shankar-Hari M, Annane D, Bauer $M$, et al. The third international consensus definitions for sepsis and septic shock (Sepsis-3). JAMA. 2016;315(8):801-10.

2. Klein Klouwenberg PM, Ong DS, Bonten MJ, Cremer OL. Classification of sepsis, severe sepsis and septic shock: the impact of minor variations in data capture and definition of SIRS criteria. Intensive Care Med. 2012;38(5):811-9.

3. Levy MM, Fink MP, Marshall JC, Abraham E, Angus D, Cook D, et al. 2001 SCCM/ESICM/ACCP/ATS/SIS international sepsis definitions conference. Crit Care Med. 2003;31(4):1250-6.

4. American College of Chest Physicians/Society of Critical Care Medicine Consensus Conference: definitions for sepsis and organ failure and guidelines for the use of innovative therapies in sepsis. Crit Care Med. 1992; 20(6):864-74. https://journals.Iww.com/ccmjournal/Abstract/1992/06000/ American_College_of_Chest_Physicians_Society_of.25.aspx.

5. Levy MM, Dellinger RP, Townsend SR, Linde-Zwirble WT, Marshall JC, Bion J, et al. The surviving sepsis campaign: results of an international guidelinebased performance improvement program targeting severe sepsis. Crit Care Med. 2010;38(2):367-74.

6. Dellinger RP, Levy MM, Rhodes A, Annane D, Gerlach H, Opal SM, et al. Surviving sepsis campaign: international guidelines for management of severe sepsis and septic shock: 2012. Crit Care Med. 2013;41(2):580-637.

7. Muessig JM, Nia AM, Masyuk M, Lauten A, Sacher AL, Brenner T, et al. Clinical frailty scale (CFS) reliably stratifies octogenarians in German ICUs: a multicentre prospective cohort study. BMC Geriatr. 2018;18(1):162.

8. Verboom DM, Frencken JF, Ong DSY, Horn J, van der Poll T, Bonten MJM, et al. Robustness of sepsis-3 criteria in critically ill patients. J Intensive Care. 2019;7:46.

9. Shankar-Hari M, Harrison DA, Rubenfeld GD, Rowan K. Epidemiology of sepsis and septic shock in critical care units: comparison between sepsis-2 and sepsis-3 populations using a national critical care database. Br J Anaesth. 2017;119(4):626-36.

10. Whittaker SA, Mikkelsen ME, Gaieski DF, Koshy S, Kean C, Fuchs BD. Severe sepsis cohorts derived from claims-based strategies appear to be biased toward a more severely ill patient population. Crit Care Med. 2013;41(4):945-53.

11. Rhee C, Jentzsch MS, Kadri SS, Seymour CW, Angus DC, Murphy DJ, et al. Variation in identifying sepsis and organ dysfunction using administrative versus electronic clinical data and impact on hospital outcome comparisons. Crit Care Med. 2019;47(4):493-500.

12. Filbin MR, Lynch J, Gillingham TD, Thorsen JE, Pasakarnis CL, Nepal S, et al. Presenting symptoms independently predict mortality in septic shock: importance of a previously unmeasured confounder. Crit Care Med. 2018.

13. Driessen RGH, van de Poll MCG, Mol MF, van Mook W, Schnabel RM. The influence of a change in septic shock definitions on intensive care epidemiology and outcome: comparison of sepsis-2 and sepsis-3 definitions. Infect Dis (Lond). 2018;50(3):207-13.

14. Shankar-Hari M, Phillips GS, Levy ML, Seymour CW, Liu VX, Deutschman CS, Sepsis definitions task F, et al. Developing a new definition and assessing new clinical criteria for septic shock: for the third international consensus definitions for sepsis and septic shock (Sepsis-3). JAMA. 2016;315(8):775-87.

\section{Publisher's Note}

Springer Nature remains neutral with regard to jurisdictional claims in published maps and institutional affiliations.

\section{Ready to submit your research? Choose BMC and benefit from}

- fast, convenient online submission

- thorough peer review by experienced researchers in your field

- rapid publication on acceptance

- support for research data, including large and complex data types

- gold Open Access which fosters wider collaboration and increased citations

- maximum visibility for your research: over $100 \mathrm{M}$ website views per year

At BMC, research is always in progress.

Learn more biomedcentral.com/submissions 